\title{
A Three-Dimensional Thermal Shock Problem with Memory-Dependent Derivative and Fractional Order Strain
}

\author{
Zhang-Na Xue, Xiao-Geng Tian \\ State Key Laboratory for Strength and Vibration of Mechanical Structures, Xi'an Jiaotong University \\ 710049, Xi'an, China \\ zhangna@ualberta.ca; tiansu@mail.xjtu.edu.cn
}

\begin{abstract}
In this work, a three-dimensional model of the Lord and Shulman (L-S) generalized thermoelasticity with memorydependent derivative (MDD) and fractional order strain is established. This model is developed by extending classical thermoelasticity models in two aspects: fractional order strain is employed to depict the mechanical phenomena caused by viscoelasticity; while the memory dependence of heat conduction is considered using MDD. The effects of each parameter on the behavior of the field quantities are elucidated and represented graphically. The results show that the introduced parameters make the distribution of the studied fields smoother in comparison with the classical models.
\end{abstract}

Keywords: Generalized Thermoelasticity, Three-Dimensional Model, Memory-Dependent Derivative, Fractional Order Strain.

\section{Introduction}

In recent years, inspired by the successful applications of fractional calculus in different areas of physics and engineering, generalized thermoelasticity (GTE) models have been further extended into temporal fractional ones [1-3] to express memory-dependence in heat conductive sense. Recently, Wang and $\mathrm{Li}$ [4] proposed a memory-dependent derivative (MDD), which is defined in an integral form of a common derivative with a kernel function on a slipping interval, which is better than the fractional one in reflecting the memory effect (instantaneous change rate depends on the past state). Yu et al. [5] first introduced the MDD instead of fractional calculus into the rate of heat flux in the Lord and Shulman (L-S) theory [6]. More applications on the MDD of thermoelasticity model can be found in references [7].

On the other hand, ultrafast pulsed lasers have been widely used in engineering, especially in materials processing. Thermal stresses due to laser heating of a material play an important role in materials processing applications where the goal is to alter the microstructure of the material near its surface. A viscoelastic constitutive model is more appropriate for the preheated material in these situations since the temperature in the material adjacent to the surface is close to its melting temperature (rather than melting) [10]. In a latest work, Youssef [11] established a one-dimensional generalized thermoelasticity theory with fractional order strain. Xue et al. [12] conducted nonlocal thermoelastic analysis considering fractional order strain in multilayered structures in the one-dimensional case.

To our knowledge, till now, one-dimensional generalized thermoelastic models with MDD or fractional order strain have been applied to different problems by many researchers with different approaches. Nevertheless, hardly any attempt has been made to investigate the three-dimensional thermoelastic problems considering MDD and fractional order strain, which is very important in materials processing applications. The aim of the present work is to investigate a half-space subjected to a thermal shock under the L-S model with MDD and fractional order strain in three-dimensional. Laplace transform and double Fourier transform techniques and inverse transforms are adopted to get the solutions. Numerical results for temperature, displacement and stress distribution are presented graphically.

\section{Formulation of the Problem And Basic Equations}

Consider an isotropic, homogenous thermoelastic half-space which fills the region $R=\{x, y, z: 0 \leq x<\infty,-\infty<y<\infty,-\infty<z<\infty\}$ 
The body is initially at rest and has been thermally shocked at the bounding plane $x=0$ when this surface is assumed to be traction free. Employing the Cartesian co-ordinates $(x, y, z)$, and the components of the displacement vector are $u_{i}=(u, v, w)$. The basic equations of the theory of L-S model with MDD and fractional order strain in the absence of body forces and heat source, take the following forms:

Memory-dependent heat conduction law [5]:

$$
q_{i}+\tau_{0} D_{w} q_{i}=-k \theta_{, i}
$$

where $\tau_{0}$ is the thermal relaxation time, $q_{i}$ is the heat flux vector, $k$ is thermal conductivity, $\theta=T-T_{0}$ is the temperature increment and $T_{0}$ is the reference temperature.

The $m$ order MDD of function $f$ has the form:

$$
D_{\omega}^{m} f(t)=\frac{1}{\omega} \int_{t-\omega}^{t} K(t-\xi) f^{(m)}(\xi) d \xi
$$

of which the kernel function $K(t-\xi)$ and the time delay $\omega$ are arbitrarily chosen, as a result, providing more possibilities to capture material's real behaviors. In particular, the kernel function can be chosen as [4].

Stress-strain relation with fractional order strain [11]:

$$
\sigma_{i j}=2 \mu\left(1+\tau^{\beta} D_{t}^{\beta}\right) e_{i j}+\lambda\left(1+\tau^{\beta} D_{t}^{\beta}\right) e \delta_{i j}-\gamma \theta \delta_{i j}
$$

where $\lambda$ and $\mu$ are Lame's constant, $\gamma=(3 \lambda+2 \mu) \alpha_{t}$ in which $\alpha_{t}$ is the coefficient of linear thermal expansion, $\tau$ is defined as the mechanical relaxation time, $\beta$ is fractional order strain parameter, $\delta_{i j}$ is the Dirichlet function, $e=u_{k, k}$ is the volumetric strain.

The time fractional derivative is defined as in [13]:

$$
D_{t}^{\beta} f^{(m)}(t)=\frac{1}{\Gamma(1-\beta)} \int_{0}^{t} \frac{f^{(m)}(\xi)}{(t-\xi)^{\beta}} d \xi \quad 0 \leq \beta \leq 1
$$

Equation of motion with fractional order strain:

$$
\rho \ddot{u}_{i}=\mu\left(1+\tau^{\beta} D_{t}^{\beta}\right) u_{i, j j}+(\lambda+\mu)\left(1+\tau^{\beta} D_{t}^{\beta}\right) u_{j, j i}-\gamma \theta_{, \mathrm{i}}
$$

The heat conduction equation with MDD and fractional order strain:

$$
k \theta_{, i i}=\left(1+\tau_{0} D_{\omega}\right)\left(\rho c_{E} \dot{\theta}+\gamma T_{0}\left(1+\tau^{\beta} D_{t}^{\beta}\right) \dot{e}_{i j} \delta_{i j}\right)
$$

where $\Gamma(1-\beta)$ is the Gamma function, $u_{i}$ is the component of the displacement vector, $c_{E}$ is the heat capacity. Introduce the following non-dimensional variables: 


$$
\begin{aligned}
& \left(x^{\prime}, y^{\prime}, z^{\prime}, u^{\prime}, v^{\prime}, w^{\prime}\right)=c_{0} \eta_{0}(x, y, z, u, v, w),\left(t^{\prime}, \tau^{\prime}, \tau_{0}{ }^{\prime}\right)= \\
& c_{0}^{2} \eta_{0}\left(t, \tau, \tau_{0}\right), \theta^{\prime}=\gamma \theta /(\lambda+2 \mu), \sigma_{i j}{ }^{\prime}=\sigma_{i j} /(\lambda+2 \mu) \\
& q_{i}^{\prime}=\gamma q_{i} / k \rho c_{0}^{3} \eta_{0}, c_{0}=\sqrt{(\lambda+2 \mu) / \rho}, \eta_{0}=\rho c_{E} / k
\end{aligned}
$$

Applying the above dimensionless variables, Eq. (5) can be written as (dropping the prime for convenience):

$$
\left(1+\tau^{\beta} D_{t}^{\beta}\right) \nabla^{2} e-\nabla^{2} \theta=\ddot{e}
$$

In addition, the heat conduction equation and the stress components become:

$$
\begin{gathered}
\nabla^{2} \theta=\left(1+\tau_{0} D_{\omega}\right)\left[\dot{\theta}+\varepsilon_{0}\left(1+\tau^{\beta} D_{t}^{\beta}\right) \dot{e}\right] \\
\sigma_{x x}=2 \beta_{0}\left(1+\tau^{\beta} D_{t}^{\beta}\right) e_{x x}+\left(1-2 \beta_{0}\right)\left(1+\tau^{\beta} D_{t}^{\beta}\right) e-\theta \\
\sigma_{y y}=2 \beta_{0}\left(1+\tau^{\beta} D_{t}^{\beta}\right) e_{y y}+\left(1-2 \beta_{0}\right)\left(1+\tau^{\beta} D_{t}^{\beta}\right) e-\theta \\
\sigma_{z z}=2 \beta_{0}\left(1+\tau^{\beta} D_{t}^{\beta}\right) e_{z z}+\left(1-2 \beta_{0}\right)\left(1+\tau^{\beta} D_{t}^{\beta}\right) e-\theta
\end{gathered}
$$

where $\beta_{0}=\mu /(\lambda+2 \mu), \varepsilon_{0}=\gamma^{2} T_{0} /\left[\rho c_{E}(\lambda+2 \mu)\right]$.

By using Eqs. (9)-(11), we consider the invariant stress to be the mean value of the principal stresses as:

$$
\sigma=\alpha_{0}\left(1+\tau^{\beta} D_{t}^{\beta}\right) e-\theta
$$

where $\alpha_{0}=\left(3-4 \beta_{0}\right) / 3$.

We assume that the boundary conditions have the forms:

$$
\begin{gathered}
\sigma(0, y, z, t)=\sigma_{x x}(0, y, z, t)=\sigma_{y y}(0, y, z, t)=\sigma_{z z}(0, y, z, t)=0 \\
\theta(0, y, z, t)=\theta_{0} H(t) g(y, z)
\end{gathered}
$$

where $\theta_{0}$ is constant and $H(t)$ is the Heaviside unit step function.

Applying Laplace transform to both sides of Eqs. (7)-(11) and considering zero initial conditions, we obtain:

$$
\begin{gathered}
\left(1+\tau^{\beta} s^{\beta}\right) \nabla^{2} \bar{e}-\nabla^{2} \bar{\theta}=s^{2} \bar{e} \\
\nabla^{2} \bar{\theta}=(1+G)\left[s \bar{\theta}+\varepsilon_{0}\left(1+\tau^{\beta} s^{\beta}\right) s \bar{e}\right] \\
\bar{\sigma}_{x x}=2 \beta_{0}\left(1+\tau^{\beta} s^{\beta}\right) \bar{e}_{x x}+\left(1-2 \beta_{0}\right)\left(1+\tau^{\beta} s^{\beta}\right) \bar{e}-\bar{\theta} \\
\bar{\sigma}_{y y}=2 \beta_{0}\left(1+\tau^{\beta} s^{\beta}\right) \bar{e}_{y y}+\left(1-2 \beta_{0}\right)\left(1+\tau^{\beta} s^{\beta}\right) \bar{e}-\bar{\theta}
\end{gathered}
$$




$$
\begin{gathered}
\bar{\sigma}_{z z}=2 \beta_{0}\left(1+\tau^{\beta} s^{\beta}\right) \bar{e}_{z z}+\left(1-2 \beta_{0}\right)\left(1+\tau^{\beta} s^{\beta}\right) \bar{e}-\bar{\theta} \\
\bar{\sigma}=\alpha_{0}\left(1+\tau^{\beta} s^{\beta}\right) \bar{e}-\bar{\theta}
\end{gathered}
$$

Where $G=\frac{\tau_{0}}{\omega}\left[\left(1-e^{-s \omega}\right)\left(1-\frac{2 b}{\omega s}+\frac{2 a^{2}}{\omega^{2} s^{2}}\right)-\left(a^{2}-2 b+\frac{2 a^{2}}{\omega s}\right) e^{-s \omega}\right]$

Eliminating $\bar{e}$ among Eqs. (15), (16)and (20), we get:

$$
\begin{aligned}
& \nabla^{2} \bar{\sigma}=\alpha_{1} \bar{\theta}+\alpha_{2} \bar{\sigma} \\
& \nabla^{2} \bar{\theta}=\alpha_{3} \bar{\theta}+\alpha_{4} \bar{\sigma}
\end{aligned}
$$

Where $\alpha_{1}=s\left(\alpha_{0}-1\right)(1+G)\left(1+\varepsilon_{0} / \alpha_{0}\right)+s^{2} /\left(1+\tau^{\beta} s^{\beta}\right), \alpha_{2}=s\left(\alpha_{0}-1\right)(1+G) \varepsilon_{0} / \alpha_{0}+s^{2} /\left(1+\tau^{\beta} s^{\beta}\right)$ $\alpha_{3}=s(1+G)\left(1+\varepsilon_{0} / \alpha_{0}\right), \alpha_{4}=s(1+G) \varepsilon_{0} / \alpha_{0}$

Applying the Fourier transform to Eqs. (21) and (22), we have the following system of ordinary differential equations:

$$
\begin{aligned}
& {\left[d^{2} / d x^{2}-\beta_{1}\right] \bar{\sigma}^{*}=\alpha_{1} \bar{\theta}^{*}} \\
& {\left[d^{2} / d x^{2}-\beta_{2}\right] \bar{\theta}^{*}=\alpha_{4} \bar{\sigma}^{*}}
\end{aligned}
$$

where $\beta_{1}=q^{2}+p^{2}+\alpha_{2}$ and $\beta_{2}=q^{2}+p^{2}+\alpha_{3}$ in which $p, q$ are the Fourier variables.

By eliminating $\bar{\sigma}^{*}$ and $\bar{\theta}^{*}$, we obtain:

$$
\begin{aligned}
& {\left[d^{4} / d x^{4}-L d^{2} / d x^{2}+M\right] \bar{\theta}^{*}=0} \\
& {\left[d^{4} / d x^{4}-L d^{2} / d x^{2}+M\right] \bar{\sigma}^{*}=0}
\end{aligned}
$$

where $L=\beta_{1}+\beta_{2}$ and $M=\beta_{1} \beta_{2}-\alpha_{1} \alpha_{4}$.

Considering the boundary conditions at infinity, the general solutions of Eqs. (25) and (26) can be written as:

$$
\bar{\theta}^{*}=\sum_{i=1}^{2} A_{i} e^{-k_{i} x}, \bar{\sigma}^{*}=\sum_{i=1}^{2} B_{i}\left[k_{i}^{2}-\beta_{2}\right] e^{-k_{i} x}
$$

where $k_{i}(i=1,2)$ are positive the roots of the characteristic equation:

$$
k^{4}-L k^{2}+M=0
$$

Substituting Eq. (27) into Eq. (24), we obtain: 


$$
A_{i}=\alpha_{4} B_{i}
$$

where $B_{i}$ can be determined by the boundary conditions after the Laplace and Fourier transform:

$$
\begin{gathered}
\bar{\sigma}^{*}(0, p, q, s)=\bar{\sigma}_{x x}^{*}(0, p, q, s)=\bar{\sigma}_{y y}^{*}(0, p, q, s) \\
=\bar{\sigma}_{z z}^{*}(0, p, q, s)=0 \\
\bar{\theta}^{*}(0, p, q, s)=\theta_{0} g^{*}(p, q) / s
\end{gathered}
$$

After applying the Laplace and Fourier transform to Eq. (5), we can get the displacement:

$$
\left(\frac{d^{2}}{d x^{2}}-k_{3}^{2}\right) \bar{u}^{*}=\frac{1}{\beta_{0}\left(1+\tau^{\beta} s^{\beta}\right)} \frac{\partial \bar{\theta}^{*}}{\partial x}-\frac{1-\beta_{0}}{\beta_{0}} \frac{\partial \bar{e}^{*}}{\partial x}
$$

where $k_{3}^{2}=p^{2}+q^{2}+s^{2} /\left[\beta_{0}\left(1+\tau^{\beta} s^{\beta}\right)\right]$.

Substituting Eqs. (27) and (29) into Eq. (32), we get:

$$
\left(d^{2} / d x^{2}-k_{3}^{2}\right) \bar{u}^{*}=L_{1} e^{-k_{1} x}+L_{2} e^{-k_{2} x}
$$

where $L_{1}=k_{1}\left[\left(1-\beta_{0}\right)\left(k_{1}^{2}-\beta_{2}+\alpha_{4}\right)-\alpha_{0} \alpha_{4}\right] B_{1} /\left[\alpha_{0} \beta_{0}\left(1+\tau^{\beta} s^{\beta}\right)\right], L_{2}=k_{2}\left[\left(1-\beta_{0}\right)\left(k_{2}^{2}-\beta_{2}+\alpha_{4}\right)-\alpha_{0} \alpha_{4}\right] B_{2} /\left[\alpha_{0} \beta_{0}\left(1+\tau^{\beta} s^{\beta}\right)\right]$

The general solution of Eq. (33) takes the following form:

$$
\bar{u}^{*}=C e^{-k_{3} x}+\frac{L_{1}}{k_{1}^{2}-k_{3}^{2}} e^{-k_{1} x}+\frac{L_{2}}{k_{2}^{2}-k_{3}^{2}} e^{-k_{2} x}
$$

where $k_{1}^{2} \neq k_{2}^{2} \neq k_{3}^{2}$ and $C$ is constant to be determined.

Considering Eqs. (17) and (20), after using the double Fourier transforms we have:

$$
\bar{\sigma}_{x x}^{*}=2 \beta_{0}\left(1+\tau^{\beta} s^{\beta}\right) \frac{\partial \bar{u}^{*}}{\partial x}+\frac{1-2 \beta_{0}}{\alpha_{0}} \bar{\sigma}^{*}+\left[\frac{1-2 \beta_{0}}{\alpha_{0}}-1\right] \bar{\theta}^{*}
$$

By using the boundary conditions (30), we get:

$$
C=\frac{1}{k_{3}}\left[\frac{\left(1-\alpha_{0}-2 \beta_{0}\right)\left(B_{1}+B_{2}\right) \alpha_{4}}{2 \alpha_{0} \beta_{0}\left(1+\tau^{\beta} s^{\beta}\right)}-\frac{L_{1} k_{1}}{k_{1}^{2}-k_{3}^{2}}-\frac{L_{2} k_{2}}{k_{2}^{2}-k_{3}^{2}}\right]
$$

In the same manner, we can get the other components of displacement. 
To obtain the solutions of the problem in the physical domain, inverse Laplace and double Fourier transforms should be conducted. Firstly, we invert the double Fourier transform using the inversion formula in the literature. Then, the inverse Laplace proposed by Brancik [14] is used.

\section{Numerical Results and Discussion}

In this section, we aim at illustrating the numerical results of the analytical expressions obtained in the previous section and elucidating the influence of time-delay, and mechanical relaxation time on the behavior of the field quantities.

Here, the function $g(y, z)=H(c-|y|) H(c-|z|)$ and $\bar{g}^{*}(p, q)=2 \sin (p c) \sin (q c) /(\pi p q)$, in which $c$ is constant. This means that the thermal shock is acting on a band of width $2 c$ centered around the $y$-axis and $z$-axis on the surface of the half space $(x=0)$.

The calculations are performed for $\theta_{0}=1, c=0.1, \tau_{0}=0.05$, and $t=0.05$. The copper material is chosen for numerical evaluations with the physical constants used in [12]. It is worth noting that in the following calculations the kernel function is assumed to be $1-(t-\xi) / \omega$.

Firstly, we consider the effects of time-delay on the response when $\tau=0.02$ and $\beta=0.5$, as shown in Figs. 1-3. It is observed from Fig. 1 that the time-delay has great effects on the temperature: the larger the time-delay, the smoother the temperature distribution, which means the particles transport the heat to the other particles easily and this makes the temperature decreases faster than the other ones. Fig. 2 indicates that although very small, the displacement is also affected by the time-delay due to the change of the temperature. One may get from Fig. 3 that the stress field has the same behavior as the temperature, that is, the distribution of stress becomes smoother as the time-delay increases. In addition, we can also find that the values of temperature, displacement, and the magnitude of stress are higher for $y=z=0$ than for $y=z=0.1$, which may be due to the fact that the position $y=z=0$ is adiabatic owing to the symmetrical condition, whilst the heat can still pass around from other locations.

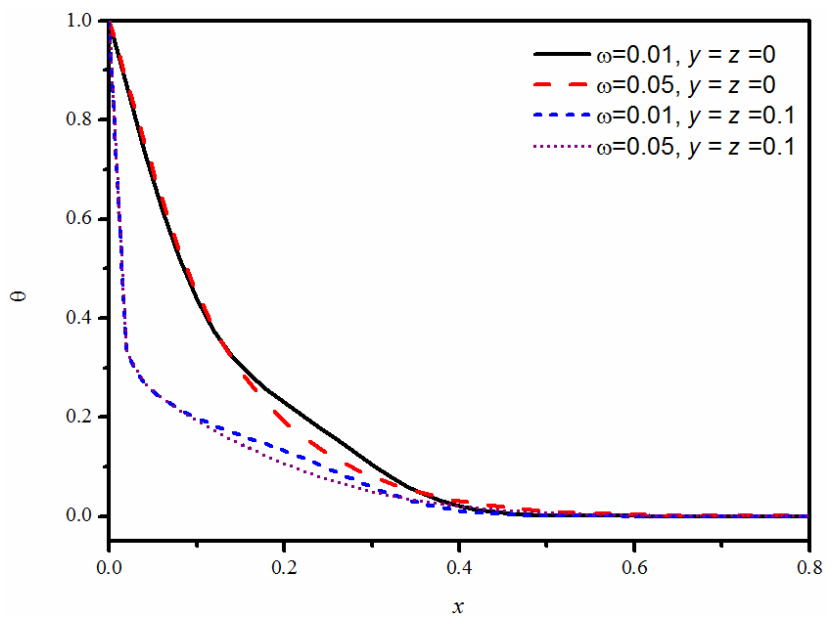

Fig. 1: The temperature distribution for different values of $y, z$ and $\omega$. 


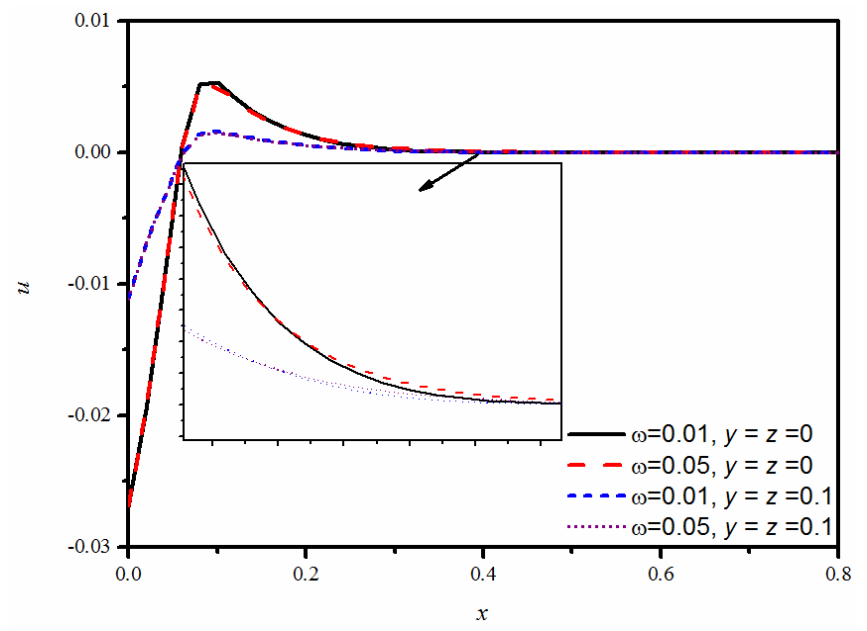

Fig. 2: The displacement distribution for different values of $y, z$ and $\omega$.

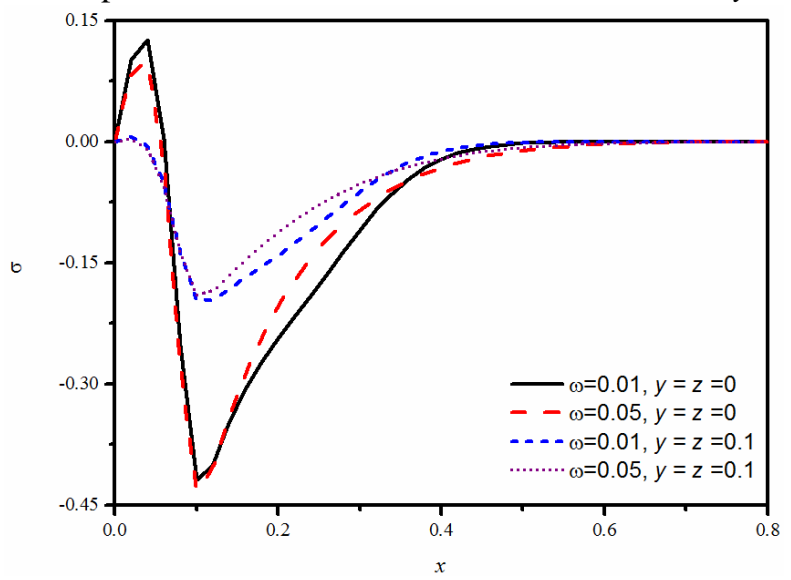

Fig. 3: The stress distribution for different values of $y, z$ and $\omega$.

The effects of mechanical relaxation time on the responses are given in Figs. 4- 6 with $\omega=0.05$ and $\beta=0.5$. It can be seen that the introduction of the mechanical relaxation time eliminates the discontinuities and sharp points in the distribution of displacement and stress. Although the mechanical relaxation time has great effects on the displacement and stress, the non-dimensional displacement is on the order of $10^{-2}$, which may further induce temperature change on the same order, i.e. $10^{-2}$. The influence is much smaller than that of the temperature change on the boundary, i.e. on the order of $10^{0}$, resulting in little influence on the temperature.

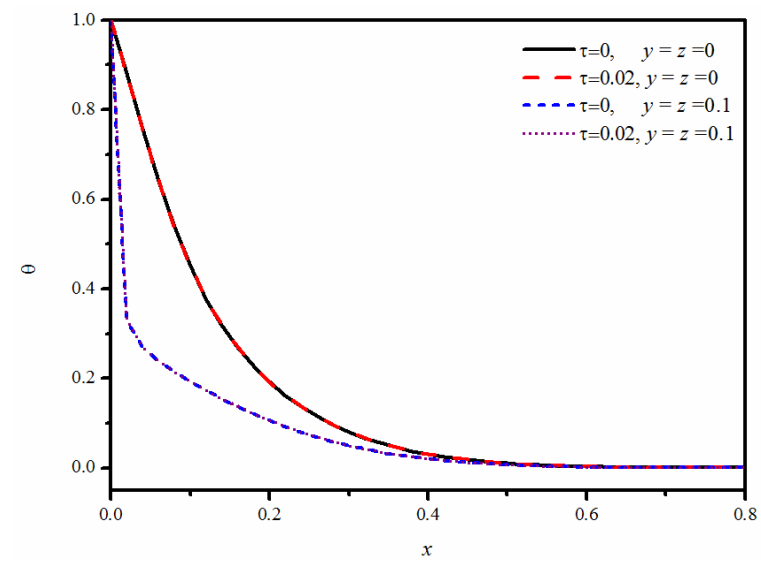


Fig. 4: The temperature distribution for different values of $y, z$ and $\tau$.

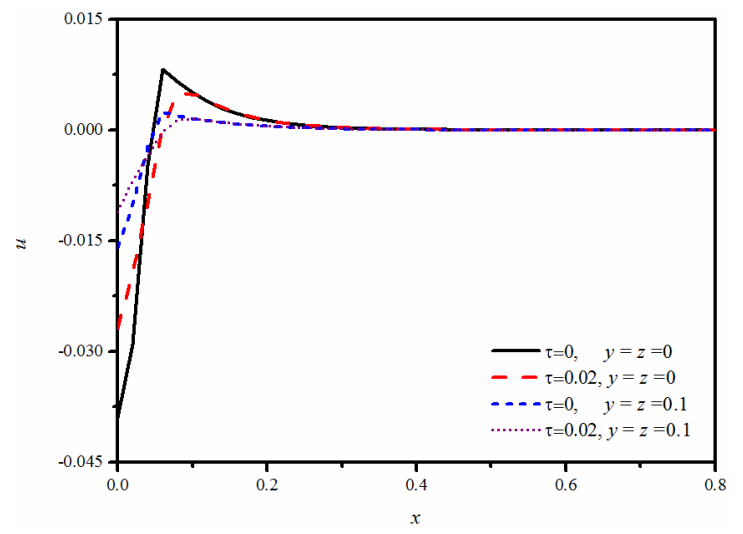

Fig. 5: The displacement distribution for different values of $y, z$ and $\tau$.

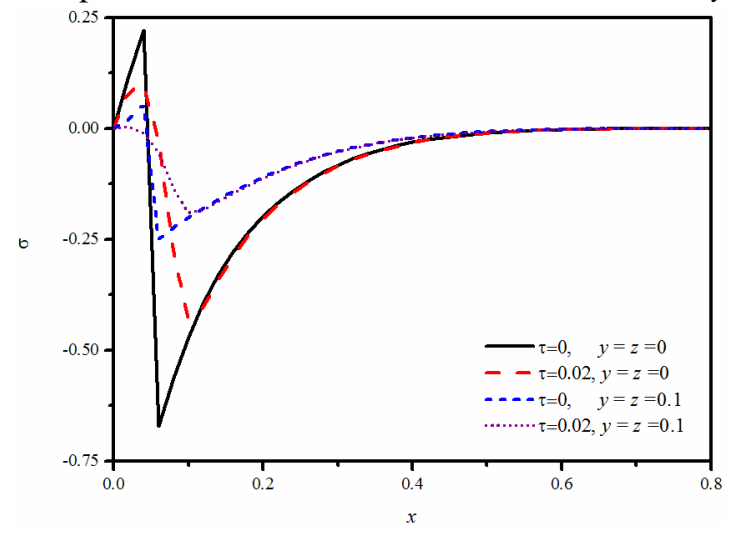

Fig. 6: The stress distribution for different values of $y, z$ and $\tau$.

Also, the results may be presented under different kernel function and fractional order strain parameters to investigate the effects of each parameter, while here we would like to cross this step.

\section{Conclusion}

A three-dimensional model of the L-S generalized thermoelasticity with MDD and fractional order strain is established and the study shows that time-delay and mechanical relaxation time have great effects on the thermoelastic responses. The introduction of these parameters makes the discontinuities and sharp points of studied fields disappear, resulting in the curves being smoothed. These findings lay the ground for further applications of ultrafast lasers in materials processing applications.materials processing applications.

\section{References}

[1] Y. Z. Povstenko, "Fractional heat conduction equation and associated thermal stress," J. Therm. Stress., vol. 28, pp. 83-102, 2004.

[2] H. H. Sherief, A. M. A. El-Sayed and A. M. Abd El-Latief, "Fractional order theory of thermoelasticity," Int. J. Solids Struct., vol. 47, no. 2, pp. 269-275, 2010.

[3] H. M. Youssef, "Theory of fractional order generalized thermoelasticity," J. Heat Trans.-T ASME, vol. 132, pp. 061301-1-7, 2010.

[4] J. L. Wang and H. F. Li, "Surpassing the fractional derivative: Concept of the memory-dependent derivative," Comput. Math. Appl., vol. 62, pp. 1562-1567, 2011. 
[5] Y. J. Yu, X. G. Tian and X. R. Liu, "Size-dependent generalized thermoelasticity using Eringen's nonlocal model," Eur. J. Mech. A/Solids, vol. 51, pp. 96-106, 2015.

[6] H. W. Lord and Y. Shulman, "A generalized dynamical theory of thermoelasticity," J. Mech. Phys. Solid., vol. 15, pp. 299-309, 1967.

[7] M. A. Ezzat, A. S. El-Karamany and A. A. El-Bary, "Generalized thermo-viscoelasticity with memory-dependent derivatives," Int. J. Mech. Sci., vol. 89, pp. 470-475, 2014.

[8] M. A. Ezzat, A. S. El Karamany and A. A. El-Bary, "Electro-thermoelasticity theory with memory-dependent derivative heat transfer," Int. J. Eng. Sci., vol. 99, pp. 22-38, 2016.

[9] A. S. El-Karamany and M. A. Ezzat, "Thermoelastic diffusion with memory-dependent derivative," J. Therm. Stress., vol. 39, pp. 1035-1050, 2016.

[10] L. G. Hector Jr. and R.B. Hetnarski, "Thermal Stresses in Materials Due to Laser Heating," in Thermal Stresses, R. B. Hetnarski, Ed. Amsterdam: Elsevier, 1996, pp. 453-466.

[11] H. M. Youssef, "Theory of Generalized Thermoelasticity with Fractional Order Strain," J. Vib. Control, vol. 22, pp. 3840-3857, 2016.

[12] Z. N. Xue, Y. J. Yu, X. Y. Li and X. G. Tian, "Nonlocal thermoelastic analysis with fractional order strain in multilayered structures," J. Therm. Stress., vol. 41, pp. 80-97, 2018.

[13] Podlubny, Fractional Differential Equations: An Introduction to Fractional Derivatives, Fractional Differential Equations, to Methods of their Solution and some of their Applications. New York: Academic Press, 1998.

[14] L. Brancik, "Programs for fast numerical inversion of Laplace transforms in MATLAB language environment," in Proceedings of the 7th conference MATLAB'99, Prague, 1999, pp. 27-39. 